\title{
Halbwachs vs Durkheim. Une critique de l'individu pur (et de la sociologie)
}

\section{Annette Disselkamp}

\section{(2) OpenEdition}

12 Journals

Édition électronique

URL : https://journals.openedition.org/ress/469

DOI : 10.4000/ress.469

ISSN : 1663-4446

Éditeur

Librairie Droz

Édition imprimée

Date de publication : 1 février 2005

Pagination : 115-133

ISBN : 2-600-00958-2

ISSN : 0048-8046

Référence électronique

Annette Disselkamp, « Halbwachs vs Durkheim. Une critique de l'individu pur (et de la sociologie) », Revue européenne des sciences sociales [En ligne], XLIII-131 | 2005, mis en ligne le 12 novembre 2009, consulté le 21 septembre 2021. URL : http://journals.openedition.org/ress/469 ; DOI : https://doi.org/ $10.4000 /$ ress.469 
Annette DISSELKAMP

\section{HALBWACHS VS DURKHEIM. UNE CRITIQUE DE L'INDIVIDU PUR (ET DE LA SOCIOLOGIE)}

\section{INTRODUCTION}

Il est bien connu qu'Emile Durkheim a plus que tout autre décrit et analysé l'organisation propre du monde social. En revanche, on s'est moins intéressé à l'autre face de sa pensée, qui concerne la conception de l'individu. De fait, la théorie que la sociologie a pour objet un ordre de manifestations spécifique, non réductible à l'expression personnelle, a pour contrepartie, chez Durkheim, la vision en vertu de laquelle les phénomènes privés constituent, de leur côté, des faits sui generis: tantôt insatiable, tantôt content de peu de choses, mû par des motifs spécifiques et replié sur lui-même, la personne particulière se situe en dehors de la vie collective. Comme la notion durkheimienne du fait social prend tout son sens dans cette opposition à l'individualité, on peut aller jusqu'à affirmer que la sociologie se met en place contre l'idée d'homme isolé abstrait'.

Cette situation représente un défi pour les collaborateurs et successeurs de Durkheim. Leur tâche consiste désormais à rattraper et intégrer cette individualité, qui semble se jouer de la science sociale justement parce qu'elle fait partie de ses présupposés, tout en demeurant obscure et inexpliquée. L'enjeu est de taille: étant à la fois présent et absent, l'individu risque de compromettre la mise en place de la nouvelle discipline, au moment précis où celle-ci prend forme.

L'œuvre de Maurice Halbwachs fournit l'exemple d'un tel effort consistant à récupérer l'individu, et donc à redéfinir les présuppositions essentielles de la sociologie ${ }^{2}$. Ainsi qu'il a souvent été remarqué, Halbwachs s'abstient, la plupart du temps, d'adresser à Durkheim des critiques explicites; la seule exception est constituée par Les causes du suicide. On sait cependant que, dans la totalité de ses travaux, il propose une approche sociologique renouvelée. Dans le présent article, je voudrais montrer comment il s'en prend, de façon inattendue et presque subreptice, à la conception présociale de l'homme particulier, et en vient à décrire une démarche sociologique en rupture avec celle de Durkheim. Ma démonstration laissera en grande partie de côté les travaux de Halbwachs sur la mémoire

C'est S. Lukes, Emile Durkheim. His Life and Work. A Historical and Critical Study, Stanford (Californie), Stanford University Press, 2e éd., avec une nouvelle préface de l'auteur, 1985 [1973], pp. 16-30, qui a le mieux décrit cet aspect de la pensée durkheimienne.

2 L'analyse de B. Karsenti, L' homme total. Sociologie, anthropologie et philosophie chez Marcel Mauss, Paris, PUF, 1997, pp.19-62, révèle que M. Mauss a été confronté au même problème. 
collective, dont l'originalité est frappante ${ }^{3}$; je me servirai plutôt de passages dont les thèmes et les analyses sont plus conventionnels, d'apparence en tout cas, et ce en procédant à ce que l'on pourrait appeler une micro-analyse de textes, c'est-àdire en examinant, dans le détail de chaque mot, des paragraphes sélectionnés.

Les points suivants seront relevés:

1. Selon Halbwachs, la position de Durkheim aboutit à la création d'un abîme entre les phénomènes observés, de type «individuel», d'un côté, et les faits sociaux, de l'autre. Mais en même temps, comme il n'existe aucun critère permettant de définir la limite entre les deux sphères, la science sociale, aux bases incertaines, se trouve à la fin dépourvue d'objet propre ${ }^{4}$.

2. La sociologie durkheimienne implique la description abstraite des caractéristiques « individuelles »: l'homme isolé est, selon Durkheim, ou bien insatiable, ou bien facilement satisfait. A l'aide de textes de Halbwachs, on mettra à jour les incohérences qu'entraîne l'intégration de cette représentation-là à l'analyse des phénomènes sociaux.

3. Durkheim dote l'individu de talents personnels. Ceux-ci se trouvent au principe de la division du travail. Halbwachs, sans nier l'existence de dispositions individuelles plus ou moins marquées, montre cependant qu'il relève de la spéculation d'inclure celles-ci dans l'investigation sociologique.

4. Comme on le sait, Durkheim affirme que le fait social doit être considéré comme une chose; il s'ensuit que le fait individuel sera traité de la même manière. Mais aux yeux de Halbwachs, cette vision, plutôt que de permettre de dépasser la connaissance intuitive et spontanée de la société, l'entérines.

Dans cette confrontation, on reconnaîtra, aux écrits de Halbwachs, une grande finesse conceptuelle. La démarche de la micro-analyse révèle comment ce dernier parvient, aussi efficacement que discrètement, à éviter une série de difficultés dont s'embarrasse Durkheim, et elle jette une nouvelle lumière sur les divergences entre les auteurs.

3 Comme le montre G. Namer, Postface, in M. Halbwachs, La mémoire collective, Paris, Ed. A. Michel, coll. Bibliothèque de l'Evolution de l'Humanité, 1997, pp. 239-295.

4 Ce thème doit évidemment être articulé à la question du rapport entre la sociologie et la psychologie, dont L. Mucchielli, parmi d'autres, a traité (Pour une psychologie collective: l' héritage durkheimien d'Halbwachs et sa rivalité avec Blondel durant l'entre-deux-guerres, in «Revue d'histoire des sciences humaines », 1, 1999, pp. 103-140).

5 Dans le cadre de cet article, il ne s'agit pas d'exposer, de façon exhaustive, tous les aspects de la question de l'individu chez Durkheim, ni non plus de montrer comment lui-même problématise et affine ses propres concepts (ainsi que J.-M. de Queiroz, Durkheim, le dualisme et l'individu, in «L'Année sociologique», vol. 49, 1999, pp. 109-130, l'a fait récemment, de façon très convaincante). Comme je voudrais présenter certains traits de la position durkheimienne tels qu'il apparaissent à la lumière du travail de Halbwachs, je me référerai plutôt aux passages où l'opposition fait social/ fait individuel s'exprime de façon particulièrement franche, surtout Le suicide. 


\section{DU LIEN ENTRE LE FAIT OBSERVÉ ET LE FAIT SOCIAL}

Au niveau épistémologique, le dualisme des dimensions sociale et individuelle, tel que Durkheim le conçoit ${ }^{6}$, aboutit à une difficulté d'envergure. En effet, de façon évidente, l'observation empirique a affaire à des manifestations particulières, puisque, ce que nous enregistrons immédiatement, ce n'est pas tant l'état collectif que le comportement de chaque personne. Par exemple, sur le plan concret, il existe des suicides mais non le suicide; le fait social, lui, est inaccessible aux sens. Mais quel est alors le rapport entre l'observation directe et l'analyse scientifique?

Afin de répondre au problème consistant à atteindre la réalité sociale, Durkheim affirme, au début des Règles, que la sociologie doit recourir à des «artifices de méthode». C'est la statistique qui constitue la démarche par excellence pour parvenir à séparer la dimension sociale du reste; son avantage consiste en ce qu'elle fait d'emblée abstraction de l'ordre individuel puisqu'elle «comprend tous les cas particuliers indistinctement». Le niveau social sera «isolé» de façon à ce que «les circonstances individuelles qui peuvent avoir quelque part dans la production du phénomène s'y neutralisent mutuellement et, par suite, ne contribuent pas à le déterminer »; les phénomènes sociaux apparaissent alors, «débarrassés de tout élément étranger ${ }^{7}$.

La méthode décrite dans les Règles prend, dans le Suicide, la forme spécifique d'une séparation entre les «causes sociales» et les «motifs individuels». Seules les forces collectives (l'anomie, l'égoïsme, l'altruisme) doivent être considérées comme étant les véritables facteurs responsables du courant suicidaire, tandis que les motifs particuliers ne représentent que des «prétextes» ou des «occasions», sans rapport avec les faits réellement déterminants. Par exemple, la déception, le chagrin, les revers de fortune, ou encore la maladie et la douleur, ne sont pas les vraies raisons du suicide.

Dans Les causes du suicide, Halbwachs critique, plus ouvertement qu'il ne le fait ailleurs, la démarche de Durkheim consistant à opposer l'examen du social à celui de la sphère individuelle ${ }^{8}$. Pour lui, la création d'un «abîme» entre les

- Les interprètes de Durkheim sont divisés concernant la présence du dualisme à travers toute son œuvre; je me rangerai plutôt du côté de la thèse «continuiste» (avec De Queiroz, op.cit., p. 110). $\mathrm{S}$. Lukes décrit, de façon systématique, le dualisme durkheimien, et tire l'attention sur les difficultés que celui-ci suscite. Il souligne en particulier trois aspects problématiques, à savoir premièrement, le caractère équivoque des concepts du social et de l'individuel, deuxièmement, l'absence de critère certain permettant de définir avec exactitude ce qui relève de l'une ou l'autre dimensions, et troisièmement, le fait que la distinction méthodologique soit basée sur une différenciation d'ordre ontologique (Lukes, op. cit., pp. 16-30).

E. Durkheim, Les règles de la méthode sociologique, Paris, PUF, coll. Quadrige, 1996 [1895], p. 10. Dans certaines circonstances, la tâche est simple, à savoir quand le fait collectif se présente d'emblée sous une forme indépendante de l'expression personnelle, comme le font la loi, les dogmes religieux, ou encore les règles de grammaire. Les manières sociales d'agir, de penser et de sentir «prennent [alors] un corps, une forme sensible qui leur est propre», elles se matérialisent et se « condensent». Dans ces cas-là, le fait social «est immédiatement donné à l'observation» (ibid., p. 9).

Que ce soit là la perspective de l'ouvrage, ceci est aujourd'hui généralement reconnu. Cf. par exemple S. Paugam, Préface, in M. Halbwachs, Les causes du suicide, Paris, PUF, coll. Le lien social, 2002 [1930], pp. IX-XXXVI. 
«grandes forces collectives» et les «motifs »" est de nature à soulever des problèmes épistémologiques considérables. En effet, dans la mesure où la relation entre l'acte individuel, d'un côté, et l'analyse sociologique, de l'autre, se trouve dès lors livrée à l'arbitraire - à un fait social déterminé pourra correspondre n'importe quelle expression particulière - tout lien entre l'observation et la dimension sociale semble être rompu, ce qui est naturellement absurde.

Mais avant d'introduire les considérations critiques de Halbwachs à cet égard, il convient de rappeler que Durkheim a été lui-même conscient de la difficulté, et qu'il a tenté d'y répondre, en allant au-delà des indications contenues dans les Règles, dans un chapitre très rarement cité du Suicide, intitulé «Formes individuelles des différents types de suicides ${ }^{10}$. Une personne déterminée à se donner la mort ne s'exclamera pas qu'elle s'en va parce que la société est anomique ou égoïste, dérégulée ou désintégrée: mais alors, comment les formes du suicide s'expriment-t-elles concrètement? La question du lien entre le fait social et les manifestations visibles est ici abordée de façon explicite: «on voudrait pouvoir saisir [les différents types de suicide] directement par l'observation... On voudrait voir les caractères des suicides particuliers se grouper eux-mêmes en classes distinctes, correspondant aux types qui viennent d'être distingués. De cette façon, on suivrait la diversité des courants suicidogènes depuis leurs origines sociales jusqu'à leurs manifestations individuelles ${ }^{11}$.

Durkheim essaye de résoudre cette difficulté en affirmant qu'il est possible de faire correspondre, à chaque type de suicide, une attitude mentale ou une humeur déterminées: au suicide égoïste, l'apathie, au suicide altruiste, l'énergie passionnelle ou volontaire, au suicide anomique enfin, l'irritation et le dégoût; un tableau systématique placé à la fin du chapitre résume bien ces corrélations ${ }^{12}$. On y voit comment le fait social «s'individualise dans les cas particuliers», ainsi que Durkheim le précise à propos du suicide égoïste par exemple: «cette paresse à l'action, ce détachement mélancolique résultent de cet état d'individuation exagérée par lequel nous avons défini ce type de suicide ${ }^{13}$.

Il apparaît, à travers ces remarques, que Durkheim perçoit clairement le problème consistant à donner aux recherches sociologiques «un caractère plus concret» et à relier les formes sociales «plus étroitement aux données de l'observation sensible et aux détails de l'expérience journalière». Mais l'explication qu'il fournit n'est apparemment pas suffisante, aux yeux de Halbwachs, pour résoudre le problème de la relation entre les motifs particuliers et les causes sociales. Et il est vrai que les attitudes mentales qui viennent d'être énumérées, et qui représentent, pour Durkheim, à la fois l'«expression individuelle» et la « conséquence» de l'état social, sont quant à elles détachées du reste des circonstances, comme il ressort de la phrase suivante: «sans doute, on ne peut déduire ainsi toutes les particularités que [le suicide] est susceptible de présenter; car il doit y en avoir qui dépendent de la nature propre du sujet. Chaque suicidé donne

\footnotetext{
9 M. Halbwachs, Les causes du suicide, op. cit., p. 10.

${ }^{10}$ E. Durkheim, Le suicide, Paris, PUF, coll. Quadrige, 1983 [1897], pp. 312-332.

11 Ibid., p. 312.

12 Ibid., p. 332.

${ }_{13}$ Ibid., p.317.
} 
à son acte une empreinte personnelle qui exprime son tempérament, les conditions spéciales où il se trouve et qui, par conséquent, ne peut être expliquée par les causes sociales et générales du phénomène $»^{14}$. En suivant ces indications, on se rend compte que la difficulté, bien qu'elle soit clairement évoquée par Durkheim, ne se trouve à la fin que repoussée. Tout en associant, à chaque forme de suicide, une «tonalité » avec des expressions concrètes, ce dernier continue de maintenir qu'un grand nombre de traits particuliers n'ont rien à voir avec la forme collective. L'abîme reste entier.

Halbwachs oppose, à la position durkheimienne, l'argument suivant. D'après lui, «motifs» et «causes» coïncident; on constate en effet que la situation concrète qui pousse une personne à se donner la mort est telle qu'elle «la détache ou l'exclut du milieu social, et lui impose le sentiment insupportable de sa solitude $»^{15}$. Les 《circonstances » ne méritent donc pas vraiment ce nom, puisqu'elles ne sont pas distribuées au hasard mais qu'elles possèdent une certaine régularité ainsi que Durkheim le savait au demeurant, puisqu'il écrit que les malheurs ont bien tendance à retourner «selon des proportions constantes ${ }^{16}$. La déconnexion qu'il opère entre les événements individuels et le taux social du suicide est d'autant plus frappante.

Par quel extraordinaire l'acte de ce suicider serait-il donc relié à la dimension sociale, tandis que la réflexion qui le précède immédiatement en demeurerait exclue? Halbwachs, lui, insiste bien sur ce que, «dans cette sorte d'antichambre où [le suicidaire] séjourne durant un temps plus ou moins court, entre les hommes qu'il a déjà quittés et la mort dont il va s'approcher, il conserve cependant sa nature d'être social, il retourne dans son esprit des pensées qui lui viennent du groupe, et sa volonté reste ce qu'elle était [sc. imprégné par le groupe] ${ }^{17}$. Ce n'est pas parce que la pensée subjective ne peut servir à rendre compte des phénomènes sociaux, à titre de cause, qu'elle appartient à un ordre différent et constitue une réalité à part ${ }^{18}$.

Grâce au commentaire de Halbwachs, on s'aperçoit combien est artificielle l'entreprise de séparer le cercle des phénomènes collectifs du privé, en leur accordant un statut ontologiquement différent. Ceci ne signifie pas que le point de vue individuel soit en lui-même vain, mais qu'il est insensé de faire la part de l' «individuel » et du «social », et de classer les phénomènes selon qu'ils appartiennent à l'une ou l'autre dimensions, en établissant entre eux un rapport de concurrence. Une telle tentative met en danger l'approche sociologique à sa racine: comme il est impossible de fournir un critère permettant de déterminer où s'arrête l'indivi-

14 Ibid., pp. 312-313.

15 Halbwachs, Les causes du suicide, op. cit., p. 11.

16 Durkheim, Les suicide, op. cit., p. 344; cf. aussi pp. 144-146.

17 Halbwachs, Les causes du suicide, op. cit., p. 343.

18 On peut comprendre en ce sens les observations que Halbwachs formule à l'égard de la définition du suicide chez Durkheim, en vertu de laquelle, comme l' «intention » ou la «volonté» du sujet ne sont pas à prendre en compte, le sacrifice ne peut être distingué du suicide. Or, remarque Halbwachs, cette définition est absurde, et révèle encore, s'il en était besoin, le caractère fragile de l'analyse durkheimienne, qui exclut, en les mettant sur un plan différent, les pensées privées (cf. ibid., pp. 339-360). 
duel et où commence le collectif, rien n'empêche en effet que la première qualification ne vienne occuper le domaine des phénomènes prétendument «sociaux ».

Halbwachs introduit, dans Les causes du suicide, la notion de «genre de vie » afin de rendre compte du phénomène social du suicide. Selon lui, celle-ci, définie comme «un ensemble de coutumes, de croyances et de manières d'être, qui résulte des occupations habituelles des hommes et de leur mode d'établissement ${ }^{19}$, a l'avantage d'intégrer toutes les expressions que Durkheim considérait comme de simples «motifs », et, dans le même temps, de ne pas isoler, les uns par rapport aux autres, les causes déterminantes, comme le fait ce dernier lorsqu'il étudie par exemple un à un les facteurs familial et religieux ${ }^{20}$.

A la lumière des remarques précédentes, il apparaît que l'étude de Halbwachs, loin de marquer un simple déplacement par rapport à Durkheim, propose une approche originale. Lecteur de Leibniz, Halbwachs est conscient que la question de 1 '«individuel» et du «général » ${ }^{21}$ appartient à la philosophie de la connaissance, et ne saurait fonder une discipline scientifique. Dans cette perspective, les phénomènes qui apparaissent comme «individuels», à travers les catégories durkheimiennes, relèvent simplement, pour Halbwachs, d'une autre généralité 22 .

On peut encore reprendre les réflexions de Halbwachs de la manière suivante. Si les deux niveaux, celui de l'individuel et celui du social, sont étrangers l'un à l'autre, quelle est alors la perspective juste? Du point de vue de l'analyse durkheimienne, le premier relève du préjugé ${ }^{23}$; mais à quel titre le second, plutôt que le premier, porterait-il en lui-même la garantie de la vérité?

\section{LES HOMMES SONT :}

\section{a) peu exigeants...}

Lorsqu'on sépare les dimensions du social et du particulier, il s'ensuit que les caractéristiques individuelles peuvent être constatées et décrites, autrement dit, que l'individu possède, non moins que le collectif, des attributs déterminés. En effet, la perspective durkheimienne n'a de sens que si le comportement des individus est quant à lui pourvu de qualités sui generis. La question est de savoir, desquelles?

On observe d'emblée que la «nature individuelle» se confond en grande partie, chez Durkheim, avec la «nature» tout court. C'est en tout cas ce qui ressort

19 Ibid., p. 376.

${ }^{20}$ Selon J.-C. Marcel, Halbwachs et le suicide : de la critique de Durkheim à la fondation d' une psychologie collective, in M. Borlandi, M. Cherkaoui, Le Suicide un siècle après Durkheim, Paris, PUF, coll. Sociologies, p. 183, c'est grâce à ce concept que Halbwachs achève la rupture avec le «durkheimisme orthodoxe».

${ }^{21} \mathrm{Au}$ premier chapitre des Règles, Durkheim privilégie le terme de «collectif» à celui de «général», afin de pouvoir distinguer les faits sociaux des faits biologiques et psychologiques (Durkheim, Les règles..., op. cit., p. 10). Cependant, au chapitre III, il réintroduit l'adjectif qualificatif de «général » comme critère de la «normalité», par opposition au «pathologique » (ibid., pp. 55-56).

${ }^{22}$ Comme G. Namer, op.cit., l'a montré, ce thème sera exploité plus loin dans La mémoire collective.

${ }^{23}$ Comme Durkheim l'affirme dans Les règles ..., op. cit., pp. 15-19. 
du Suicide, où ce dernier mobilise, concernant la question soulevée, des traditions philosophiques anciennes. Il impute aux hommes, tantôt le simple souci de l'autoconservation, tantôt l'illimitation des pulsions, en opposant, dans une perspective dualiste, les tendances mentionnées à la vie sociale. On peut, sans aucun doute, retracer les racines de cette vision jusqu'à la pensée antique. Mais dans le cadre de la contribution présente, nous devons nous contenter de rappeler quelle est la réaction de Halbwachs à l'égard de la théorie durkheimienne.

Vers la fin du chapitre «Le suicide égoïste», dans un passage traitant de la question de savoir pourquoi le taux de suicide est moins élevé chez le groupe des femmes, des enfants et des vieillards que chez les mâles d'âge mûr, Durkheim consacre des réflexions révélatrices à la dualité humaine. En vertu de ces considérations, l'homme «physique» se caractérise par sa nature autarcique: il est sans exigences, ses besoins sont modestes, et il vit replié voire refermé sur lui-même.

D'entrée de jeu, Durkheim affirme de façon explicite le caractère double du genre humain: «...si, comme on l'a dit souvent, l'homme est double, c'est qu'à l'homme physique se surajoute l'homme social». Une précision importante suit immédiatement, à savoir que l'homme social «est le tout de l'homme civilisé » ${ }^{24}$. La société représente ainsi ce qui sépare l'homme de l'état animal. Nous sommes des personnes humaines, distinctes des animaux, ou plutôt supérieures à eux, parce que nous vivons en groupe et que la communauté nous inspire une vie morale.

La nature, elle, concerne «l'ordre des fonctions qui n'intéressent que l'individu; celles qui sont nécessaires à l'entretien de la vie physique». Cette tendance à préserver sa propre vie, assimilée à l'《égoïsme» ${ }^{25}$, ne cherche jamais à se dépasser elle-même: "puisque [les fonctions liées à la survie] sont faites uniquement pour ce but, elles sont tout ce qu'elles doivent être quand il est atteint». L'état social, au contraire, repose sur l'ouverture envers d'autres fins et d'autres objectifs, ceux qui sont déconnectés du seul besoin de survivre, comme par exemple «l'art, la morale, la religion, la foi politique, la science elle-même»; il suscite des «sentiments de sympathie et de solidarité qui nous inclinent vers autrui $»^{26}$ et dont la nature ignore l'existence.

Sur la base de ces définitions, Durkheim développe l'argument suivant concernant la catégorie des femmes, des vieillards et des enfants. Selon lui, les personnes mentionnées se trouvent, davantage que les hommes d'âge mûr, sous l'emprise de la nature physique; à l'inverse, l'aspect social n'est qu'insuffisamment développé chez eux. Autrement dit, chez les groupes en question, «l'homme physique tend à redevenir tout l'homme ${ }^{27}$. En effet, l'enfant, «naturellement égoïste ${ }^{28}$, n'est pas encore membre à part entière de la société, puisque celle-ci

${ }^{24}$ Durkheim, Le suicide, op. cit., p. 228.

${ }^{25}$ Dans L'Education morale, la tendance égoïste s'exprime de deux manières. Elle concerne, premièrement, la conservation de soi-même, en un sens physique, et deuxièmement, le fait de s'occuper de sa propre personne (E. Durkheim, L'éducation morale, Paris, PUF, coll. Quadrige, 1992 [1902/3], pp. 48-49).

26 Durkheim, Le suicide, op. cit., p. 226.

${ }^{27}$ Ibid., p. 230.

${ }^{28}$ Ibid., p. 226. 
«n'a pas eu le temps de le former à son image »" ${ }^{29}$. A l'autre extrémité de la vie, le vieillard commence à s'en isoler, «la société s'en retire», sans doute parce que les personnes vieilles sont de plus en plus préoccupées par la préservation de leur santé, ce qui absorbe alors toute leur énergie.

Durkheim en conclut que ces groupes «se suffisent davantage», c'est-à-dire qu'ils n'ont pas besoin, autant que les mâles d'âge mûr, de participer à la vie de la société; la seule condition du bonheur, chez eux, est constituée par la satisfaction des besoins primaires. Cherchant à rendre compte du faible taux de suicide chez les personnes en question, il suggère alors que celles-ci, se trouvant à l'extérieur du groupe, ne subissent pas de plein fouet les conséquences des désordres sociaux, autrement dit que les crises de la société ne sauraient les affecter. C'est que, «ayant moins besoin de se compléter par autre chose qu' [elles]-mêmes, [elles] sont aussi moins exposées à manquer de ce qui est nécessaire pour vivre ${ }^{30}$. Une véritable incapacité à être proie à la «dépression » ou à la «mélancolie» explique ainsi leur relative immunité au suicide.

Durkheim ajoute que cette propriété que possèdent les enfants, les femmes et les vieillards, de mieux résister au courant suicidaire, est exactement identique à celle que l'on peut observer chez les animaux, puisqu'elle «n'a pas d'autres causes $»^{31}$.

L'analyse proposée par Durkheim appelle évidemment une série de remarques, concernant

- son dualisme ontologique: en vertu du passage en question, la science sociologique ne traite pas de l'homme dans son intégralité, considéré sous l'angle de la vie du groupe, mais elle s'intéresse à une moitié d'homme, à savoir sa moitié sociale;

- la définition autarcique de l'individu naturel, par opposition à l'homme social : la vie sociale commence bien là où les besoins matériels sont satisfaits, c'està-dire qu'elle évolue en dehors du nécessaire ${ }^{32}$;

- les implications du rejet des vieillards, des femmes et des enfants en dehors de la société: il est manifeste que cette exclusion concerne tous ceux qui se trouvent, en fait, dans une position de faiblesse;

- sa misogynie.

29 Ibid., p. 231.

so Ibid., p. 231.

${ }^{31}$ Ibid. Cette affirmation prend une forme caricaturale à l'égard des femmes. Durkheim écrit: «Voilà enfin pourquoi il se fait que la femme peut, plus facilement que l'homme, vivre isolée. Quand on voit la veuve supporter sa condition beaucoup mieux que le veuf et rechercher le mariage avec une moindre passion, on est porté à croire que cette aptitude à se passer de la famille est une marque de supériorité; on dit que les facultés affectives de la femme, étant très intenses, trouvent aisément leur emploi en dehors du cercle domestique, tandis que son dévouement nous est indispensable pour nous aider à supporter la vie. En réalité, si elle a ce privilège, c'est que sa sensibilité est plutôt rudimentaire que très développée. Comme elle vit plus que l'homme en dehors de la vie commune, la vie commune la pénètre moins: la société lui est moins nécessaire parce qu'elle est moins imprégnée de sociabilité. Elle n'a que peu de besoins qui soient tournés de ce côté, et elle les contente à peu de frais. Avec quelques pratiques de dévotion, quelques animaux à soigner, la vieille fille a sa vie remplie» (ibid.).

32 Sur la base de cette remarque, il conviendrait de réexaminer la place de Durkheim dans la «sociologie économique». 
On ne peut les approfondir ici; dans le cadre du présent travail, je m'intéresserai à la façon dont Halbwachs met en question la détermination des groupes sociaux par leur appartenance plus ou moins importante à la vie du groupe ou à la nature. Pour cela, je me servirai d'un texte qui n'a, à première vue, rien à voir avec les thèmes traités par Durkheim, à savoir Matière et société. Cette contribution a attiré l'attention des interprètes de Halbwachs parce qu'elle expose une théorie de l'aliénation, proche de la vision marxiste, à une époque où cette idée n'est guère connue en France ${ }^{33}$. Mais dans la confrontation entre Durkheim et Halbwachs, d'autres aspects émergent, comme celui qui nous intéresse ici, concernant la dualité des hommes. Précisions encore une fois que les passages qui seront étudiés n'ont rien de polémique; il n'est pas question du suicide, et puis, les groupes sur lesquels s'appuie la démonstration de Halbwachs ne sont pas identiques à ceux que mentionne Durkheim, à l'exception des vieillards, auxquels Halbwachs fait allusion $^{34}$.

Dans les premiers paragraphes de Matière et société, Halbwachs semble, en un premier temps, adhérer à l'idée de «double nature». Il admet en effet que, «dans une conscience où prédominent les sensations qui correspondent aux faits de la nature inorganique, la place laissée aux idées qui correspondent aux personnes se trouve réduite d'autant plus que ces deux sortes de pensées ne se confondent pas, ne s'alimentent point, et ne s'entretiennent pas en mouvement par une action réciproque». Un «ralentissement» et une «paralysie partielle des fonctions de relation» semblent alors pouvoir être observés chez les «membres de la société qu'un accident quelconque, ruine, maladie, deuil, vieillesse peut-être, etc., a conduits à s'éloigner du monde, à renoncer à nombre d'occupations et de distractions qui les mettaient en rapport avec leurs semblables». La «nature» se manifeste, dans ces groupes où «le lien social est momentanément tranché», plus puissamment que chez les autres ${ }^{35}$.

Ces observations donnent l'impression de renouveler la vision dualiste de Durkheim, avec l'idée que «nature » et «société » sont inégalement distribuées, et qu'il existe des catégories de personnes qui, soustraites à l'ascendant de la société, sont davantage livrées au règne physique. Cependant, en lisant attentivement la suite du texte, on s'aperçoit que Halbwachs n'introduit l'idée de double nature que pour pouvoir mieux la réfuter.

Concernant les vieillards, il amène dès l'abord une observation tranchante. Pour lui, les personnes âgées «ne peuvent, par définition, fonder une distinction

${ }_{33}$ V. Karady, Biographie de Maurice Halbwachs, in M. Halbwachs, Classes sociales et morphologie, Paris, Les éditions de Minuit, 1972, p. 13. L'auteur souligne toutefois que la faible sociabilité des ouvriers est un thème positiviste depuis A. Compte.

${ }^{34}$ Ainsi que l'observe G. Namer, «la tactique même de Halbwachs » consiste à «feindre d'utiliser Bergson pour réfuter Bergson comme il en fera de même au passage et pour Marx, et pour Freud, et pour Max Weber» (G. Namer, Postface, op. cit., p. 299. Aux noms de Marx, Freud et Weber, il faut ajouter celui de Durkheim (ibid., p. 308). Seulement, pour ce qui est de ce dernier, le procédé est plus implicite qu'à l'égard des autres: Halbwachs critique sa pensée sans le nommer la plupart du temps. Mais, plus indirectes, ses remarques n'en sont pas moins incisives.

${ }_{35}$ M. Halbwachs, Matière et société [1920], in Classes sociales et morphologie, op. cit., pp. 6061. Halbwachs emploie le terme «inorganique» pour préparer le thème qui l'intéresse, à savoir le rapport qu'entretiennent les ouvriers avec les machines; mais dans d'autres endroits, il a également recours à la formule «nature organique» (ibid., p. 79). 
sociale $»^{36}$, sans doute parce que, considérés comme un groupe d'âge, eu égard à leurs seules caractéristiques physiques - affaiblissement des forces vitales - les vieillards ne représentent précisément pas un objet d'étude sociologique; expliquer le faible taux de suicide, chez cette catégorie de personnes, par leur «nature», ceci revient à une tautologie. Le même type d'observation pourrait évidemment s'appliquer aux femmes et aux enfants.

Afin de pouvoir juger des autres cas, on se rappellera, en un premier temps, que «n'importe quel groupe humain est en contact avec la nature matérielle ${ }^{37}$ : le fait que toutes les personnes participent à la fois de l'ordre physique et de l'ordre social, ceci constitue une évidence qui n'explique rien. Puis, l'observation s'impose que l'isolement des groupes qui vivent à l'écart de la société n'est en réalité qu'apparent, comme par exemple chez les monastiques ou les prisonniers de guerre, ou encore les paysans, ce qui signifie qu'il est absurde de comparer entre elles différentes catégories de personnes selon qu'elles appartiennent plus ou moins aux règnes «social» ou «naturel».

Halbwachs se sert, afin d'expliquer les phénomènes d'isolement, de la formule «type de vie», proche de celle de «genre de vie», en remarquant que, dans tous les cas, les différences sont de «qualité ou d'espèce» plutôt que de «degré». Les vies paysanne et citadine, par exemple, sont «toutes deux sociales au même titre». Ici se confirme l'observation dont il a été question plus haut, à savoir que la prétendue «individualité» renvoie en réalité à une autre «généralité », comme l'écrit Halbwachs: «si certains hommes et même certains groupes restent en dehors, sinon en marge, d'une société, c'est qu'ils font partie d'autres sociétés », en précisant: «et cela s'explique par une répugnance, un antagonisme, ou une incompatibilité qui met aux prises des tendances collectives opposées ${ }^{38}$. On pourrait compléter cette citation de la manière suivante: il est vain de vouloir expliquer la position marginale des groupes mentionnés grâce à l'hypothèse que les uns subissent, plus fortement que les autres, l'impact de la nature. C'est que, bien au contraire, la situation d'isolement constitue un phénomène social à part entière, qui requiert d'être ramené à des facteurs sociaux.

Notons enfin combien il est remarquable que Halbwachs n'oublie pas, parmi les facteurs de séparation, la «ruine», le «deuil» et la «maladie ${ }^{39}:$ comme on s'en souvient, ce sont là autant de phénomènes que Durkheim avait classés parmi les causes «apparentes» et «individuelles» du suicide. Pour Halbwachs, ces manifestations méritent encore d'être traitées d'un point de vue sociologique dans la mesure où aucun phénomène n'est, en lui-même, ou bien «naturel» et « individuel» ou bien «social». Tout dépend de la perspective adoptée; et s'il est vrai que la maladie fait partie de notre condition biologique, l'isolement qu'elle est susceptible d'amener n'a rien de naturel, mais renvoie en vérité à l'organisation du groupe. Les différences entre les façons de vivre ne sauraient pour cela être expliquées par la constitution double des hommes, mais bien par des causes sociales.

\footnotetext{
${ }^{36}$ Ibid., p. 61.

${ }^{37}$ Ibid., p. 58.

${ }^{38}$ Ibid., p. 62.

39 Ibid., p. 59.
} 
Par contraste, Durkheim avait justifié la position solitaire des vieillards, des femmes et des enfants par l'absence du social et l'ascendant de la nature.

Mais venons-en aux ouvriers, auxquels la contribution Matière et société est tout particulièrement consacrée. Malgré la série d'objections qui précèdent, l'idée de dualité semble, chez eux, pleinement réalisée. Cependant, j'essaierai de montrer que c'est précisément à travers la présentation de ce groupe que l'idée de double nature se trouvera le plus profondément ébranlée.

Il est, en un premier temps, aisé d'observer que les ouvriers sont davantage en rapport avec la «matière» que tous les autres membres de la société et que leurs rapports sociaux sont sous-développés: «le mécanisme de la nature entre en eux», au point que «l'activité reste organique en son principe et son détail ${ }^{40}$. L'analyse phénoménologique de leur condition est sensiblement proche de celle des femmes, des enfants et des vieillards dans le Suicide de Durkheim. On a bien affaire à un groupe qui ne participe pas pleinement à la vie sociale, et ce, comme l'écrit Halbwachs, parce que ses membres n'en possèdent «ni les qualités, ni le pouvoir»; «les sensations corporelles de toute nature» sont «intensifiées», «le sentiment des besoins purement physiques », « renforcé », et inversement, ils sont «détachés du système des tendances que détermine en nous la vie sociale ${ }^{41}$. De façon similaire, Durkheim avait soutenu que «la sensibilité des femmes est plutôt rudimentaire que très développée» et qu'elles «n'ont que peu de besoins qui soient tournés du côté [de la société]» ${ }^{42}$.

Mais ce n'est évidemment pas la «nature naturelle» qui explique la position exceptionnelle des ouvriers; celle-ci est à son tour d'origine sociale. Il est vrai que la situation est complexe, selon Halbwachs. D’un côté, dans les machines, «la société ne retrouve que ce qu'elle y a mis », et on pourrait pour cette raison croire, à première vue, que le travailleur ne subit aucun isolement et qu'il entre au contraire en relation vivante avec la vie sociale quand il manipule un appareil. Mais Halbwachs fait remarquer, non sans finesse, qu'aucune machine, pas même la plus sophistiquée, ne saurait totalement dominer la nature, et que la tâche de l'ouvrier consiste justement à appliquer sa force à ce «résidu», ce «presque rien qui ne porte pas la marque» de la société ${ }^{43}$. Autrement dit, l'intervention de l'ouvrier est nécessaire parce que le génie technique ne saurait complètement triompher de la matière; et c'est bien pour cela que le travailleur est, plus que tout autre membre de la société, tourné vers elle. Comme ses gestes acquièrent un sens, non par rapport à d'autres faits sociaux, mais à ce reste de matière qui les détermine, l'ouvrier perd toute conscience de son entourage social.

Il est vrai que Durkheim, non moins que Halbwachs, a également remarqué que la division du travail était susceptible d'isoler les ouvriers les uns par rapport aux autres ${ }^{44}$. Mais de façon significative, pour le premier, cette situation de crise doit encore être ramenée, en dernière analyse, à un déséquilibre entre la nature

40 Ibid., p. 79.

${ }^{41}$ Ibid., pp. 63-64.

${ }^{42}$ Durkheim, Le suicide, op. cit., p. 231.

${ }^{43}$ Halbwachs, Matière et société, op. cit., p. 66.

${ }^{44}$ E. Durkheim, La division du travail social, Paris, PUF, coll. Quadrige, 1983a [1893], pp. 348-349. 
innée des individus et la place que chacun occupe au sein de la sociétét ${ }^{45}$. En revanche, chez Halbwachs, la position des ouvriers sera mise en relation avec un trait spécifique de la société moderne, consistant dans le fait que «l'essentiel [y] est de produire ${ }^{46}$. Leur condition s'explique dans ce cadre uniquement. Halbwachs fait ainsi comprendre que c'est une certaine société, aux caractéristiques déterminées et à vrai dire uniques, et non n'importe laquelle qui contraint ses membres à s'isoler des autres personnes.

\section{b) ... ou insatiables}

Les propos de Halbwachs concernant la productivité peuvent être rapprochés d'une série de réflexions dont Durkheim a fait état vers la fin du chapitre «Le suicide anomique ». Ce dernier dénonce une industrie qui, « au lieu de continuer à être regardée comme un moyen en vue d'une fin qui la dépasse, est devenue la fin suprême des individus ». "Le développement de l'industrie et l'extension presque indéfinie du marché ${ }^{47}$ correspondent à un phénomène d'anomie, consistant dans le fait que les appétits des hommes se déchaînent excessivement, de façon à engendrer des états de frustration.

Mais la similitude entre Halbwachs et Durkheim s'arrête là. En effet, aux yeux du second, l'explosion des désirs renvoie encore, en dernière analyse, à un trait inhérent à la nature humaine. C'est ce qui apparaît au début du chapitre mentionné, où Durkheim attribue aux hommes une disposition innée à ne jamais se satisfaire de ce qu'ils ont et à vouloir toujours plus. On doit, selon lui, mettre cette tendance en rapport avec la réflexion: «au-delà du minimum indispensable, dont la nature est prête à se contenter quand elle procède instinctivement, la réflexion, plus éveillée, fait entrevoir des conditions meilleures, qui apparaissent comme des fins désirables et qui sollicitent l'activité». En effet, comme «la plupart des besoins [des hommes] ne sont pas... sous la dépendance du corps », ils se déconnectent des «moyens », contrairement à ce qui se passe chez l'animal, où «l'équilibre s'établit avec une spontanéité automatique ${ }^{48}$.

Pris en eux-mêmes et «en tant qu'ils dépendent de l'individu seul», les appétits des hommes sont illimités: «ni dans la constitution organique, ni dans la constitution psychologique de l'homme, on ne trouve rien qui marque un terme à de semblables penchants »; "notre sensibilité est un abîme sans fond que rien ne peut combler ${ }^{49}$. C'est la raison pour laquelle, livré à lui-même, l'homme sera continuellement insatisfait.

45 Cf. infra, chap. 3 («Les talents innés »).

${ }^{46}$ Halbwachs, Matière et société, op.cit., p. 83. On retrouve le même point de vue vers la fin de L'esquisse d'une psychologie des classes sociales: «l'évolution économique telle qu'elle s'accomplit depuis plus d'un siècle, sous le signe du capitalisme, semble ... s'être orientée surtout vers l'accroissement, vers un accroissement illimité de la production», mais «le bonheur ... ne résulte pas automatiquement de ce que les produits, la richesse s'accroissent, se multiplient ...» (M. Halbwachs, Esquisse d'une psychologie des classes sociales, avec une notice sur l'auteur par G. Friedmann, Paris, Librairie M. Rivière et Cie, 1964 [1938], pp. 236-237).

47 Durkheim, Le suicide, op. cit., p. 284.

${ }_{48}$ Ibid., pp. 272-273.

49 Ibid., p. 273. 
Ainsi, tandis qu'au chapitre «Suicide égoïste», l'individu se caractérise par sa condition quasi-biologique d'autarcie et le fait qu'il est sans exigences, au chapitre «Suicide anomique », il possède une propriété contraire, à savoir celle de se donner des objectifs de plus en plus éloignés; celle-ci «est venue rompre l'état d'équilibre dans lequel sommeillait l'animal ${ }^{50}$.

Comme on le sait, aux yeux de Durkheim, c'est la société qui a pour vocation de contenir et de modérer les passions, et par là, de rendre les hommes capables du bonheur au sens de la satisfaction. Cette fonction s'accomplit grâce à l'intégration de chaque individu à un groupe déterminé; tout le monde admet, par exemple, qu'un ouvrier ne nourrit pas les mêmes prétentions qu'un paysan, etc.

Mais lors d'une situation de crise, c'est-à-dire d'un changement important dans l'ordre social, l'équilibre se rompt; alors les individus, dépourvus de repères, sont susceptibles d'entretenir des désirs exagérés, qui ne peuvent être comblés. D'où des états de déception; d'où, à la fin, une augmentation du taux de suicide.

Les choses se présentent différemment chez Halbwachs, qui a traité de la question de la formation des besoins en plusieurs endroits ${ }^{51}$.

Que les hommes aient des désirs, ceci est sans doute constitutif de leur condition. Mais Halbwachs hésite, là encore, à introduire des réflexions concernant notre nature dans l'explication des situations concrètes. Soulignons que la différence de perspective se reflète bien dans le choix des termes: Durkheim assimile les «besoins» aux «passions, tandis que Halbwachs, lui, parle des seuls «besoins $»^{52}$.

De façon directe, il s'oppose à l'opinion des économistes classiques ${ }^{53}$; or, comme c'est si souvent le cas, ses arguments sont propres à ébranler en même temps la position de Durkheim. Halbwachs critique avec véhémence la référence à la «nature humaine »; selon lui, celle-ci, amenant la recherche dans une impasse, est impuissante à rendre compte des phénomènes sociaux, comme il le remarque de façon laconique: «Invoquera-t-on la nature humaine? Elle resterait à peu près

so $\quad$ Ibid., p. 275.

${ }^{51}$ C'est le thème de sa thèse de doctorat, La classe ouvrière et les niveaux de vie. Recherches sur la hiérarchie des besoins dans les sociétés industrielles contemporaines, Thèse pour le doctorat présentée à la Faculté des Lettres de 1'Université de Paris, Paris/ Londres/ New York, Gordon et Breach, coll. Réimpressions GIB Sciences humaines et philosophie, 1970 [1912]; Halbwachs y consacre encore L'évolution des besoins dans les classes ouvrières, Paris, F. Alcan, coll. Nouvelle Bibliothèque Economique, 1933; puis, la question des besoins se trouve également traitée dans la contribution Les besoins et les tendances dans l'économie sociale, in Classes sociales et morphologie, op.cit., pp. 27-40. Le troisième de ces textes s'intéresse à la théorie de l'économiste allemand Gustav Schmoller. Ce dernier s'oppose, à juste titre selon Halbwachs, à l'idée que la vie économique peut être ramenée à la seule poursuite d'intérêts matériels, telle que la défendent les économistes classiques; cependant Schmoller se serait arrêté à mi-chemin, puisqu'il substitue, à l'individu intéressé, l'individu historique, alors qu'il s'agirait d'introduire la dimension sociale.

52 On peut se demander si Durkheim ne confirme pas par là la position prétendument «utilitariste» qu'il entend réfuter, dans la mesure où c'est justement celle-ci qui, ignorant toute distinction entre plusieurs types de mobiles et différentes attitudes pratiques, réduit les hommes à une seule dimension.

${ }_{53}$ M. Mauss, Essai sur le don. Forme et raison de l'échange dans les sociétés archaïque, in M. Mauss, Sociologie et anthropologie, intr. par C. Lévy-Strauss, Paris, PUF, coll. Quadrige, $7^{\circ}$ éd., 1997 [1923-24], p. 272, met bien en valeur le point de vue adopté par Halbwachs. 
la même chez l'individu à travers le temps, et elle serait identique chez les divers membres d'un groupe $»^{54}$.

Mais pourquoi les besoins augmentent-ils? Où, plus concrètement, comment se fait-il que, dans des moments de crise économique, les salaires ne baissent pas? Pourquoi «les salaires ne sont-ils pas ramenés à ce qui serait tout juste nécessaire pour assurer la subsistance des ouvriers dont on a l'emploi ?» ${ }^{55}$.

Afin de répondre à ces questions, il ne suffit pas de faire appel à l'intérêt individuel. Notons que c'est là une position que Durkheim a lui-même défendue dans La division du travail social, où il avance l'idée que l'individu, pris isolément, n'est pas capable de concevoir un bonheur différent de celui qui provient de ses habitudes ${ }^{56}$. Mais le contexte n'est pas celui du Suicide; dans La division $d u$ travail social, il s'agit d'expliquer la formation de la «société organique»; en revanche, dans Le suicide, la référence à la nature est introduite afin de rendre compte de la production d'un phénomène «pathologique», ou encore d'un déséquilibre: manifestement, la notion d'individu intervient, chez Durkheim, pour analyser une situation de désordre.

Halbwachs défend l'idée que les besoins ne sont pas «isolés» mais qu'ils font partie d'un «genre de vie» ou d'un «niveau de vie»; ces concepts nous rapprochent évidemment des Causes du suicide. Il s'agit de comprendre, pour lui, que la répartition des dépenses, comprenant par exemple la nourriture, le logement, et les vêtements, est commune à un groupe donné; on ne saurait les prendre une à une, en se demandant comment chacune d'entre elles répond à une nécessité physique déterminée, mais il faut au contraire les étudier dans leurs rapports réciproques. Autrement dit, les variations de dépense n'ont rien d'automatique ${ }^{57}$. La notion de niveau de vie permet ainsi d' " envisager les besoins comme des tendances nées de la vie sociale »; elle nous amène à comprendre que c'est par rapport à la société seulement, et non en eux-mêmes, qu' «ils nous apparaîtront comme extensibles presque indéfiniment $\gg^{58}$.

Le reproche implicite adressé à Durkheim est d'avoir oublié que les hommes réels vivent en société; en effet, ainsi que nous l'avons vu, ce dernier a au contraire recours à l'idée que c'est «en eux-mêmes » que les besoins sont susceptibles de s'affranchir de toute limite.

${ }^{54}$ Halbwachs, La classe ouvrière ..., op. cit., p. 393.

ss Halbwachs, L'évolution des besoins..., op. cit., p. 142.

56 «Voilà ce qu'on oublie trop quand on compare le bonheur de nos pères avec le nôtre. On raisonne comme si tous nos plaisirs avaient pu être les leurs; alors, en songeant à tous ces raffinements de la civilisation dont nous jouissons et qu'ils ne connaissaient pas, on se sent enclin à plaindre leur sort. On oublie qu'ils n'étaient pas aptes à les goûter. Si donc ils se sont tant tourmentés pour accroître la puissance productive du travail, ce n'était pas pour conquérir des biens qui étaient pour eux sans valeur. Pour les apprécier, il leur eût fallu d'abord contracter des goûts et des habitudes qu'ils n'avaient pas, c'est-à-dire changer leur nature» (Durkheim, La division du travail social, op . cit., p. 219).

${ }_{57}$ Halbwachs, L'évolution des besoins..., op. cit., pp. 133-152.

58 Ibid., p. 152. Pour une présentation plus détaillée, on lira J.-C. Marcel, Maurice Halbwachs et les classes sociales, in «Alternatives Economiques», n² 208, nov. 2002, pp. 77-81. Marcel défend la thèse suivant laquelle la vision de Halbwachs modifie et corrige la définition de la conscience de classe telle qu'on la trouve chez Karl Marx, puisque «ce qui constitue une classe sociale, c'est moins exclusivement une place dans les rapports de production qu'un intérêt, une idéologie et des préoccupations qui en sont issus » (p. 77). 


\section{LES TALENTS INNÉS}

L'idée de nature humaine joue un rôle central dans La division du travail social. Elle prend, dans cet ouvrage, la forme particulière du concept de don individuel: chacun est doté d'une «nature personnelle» ou «individuelle»; il existe des «aptitudes » aux différents «genres d'activité», ou encore une «diversité des capacités » et des $«$ facultés $»^{59}$.

L'idée de prédisposition individuelle correspond évidemment à un problème qui n'a jamais cessé de préoccuper la sociologie, celui de rendre compte des différences sociales ou de justifier les inégalités. La réponse à cette question est pour Durkheim inséparable de la référence aux variations naturelles. Il est remarquable que ce type d'explication se transforme en légitimation; en effet, la division du travail représente, à ses yeux, la condition du bonheur individuel, puisque, permettant à chacun d'apprendre le métier qui correspond le mieux à ses facultés innées, elle offre aux hommes une chance inouïe d'épanouissement personnel. A l'inverse, ainsi que nous l'avons mentionné plus haut, il y a déséquilibre lorsque la profession qu'une personne exerce n'est pas celle à laquelle l'appellent ses talents.

Halbwachs formule une double critique à l'égard de cette vision. Premièrement, confrontée à la réalité d'un monde du travail qui distribue, aux ouvriers, des tâches de plus en plus répétitives et abrutissantes, la formulation de Durkheim ressemble plutôt à un euphémisme. Dans Matière et société, au sein d'un passage dont il a déjà été question précédemment, à propos de la condition des travailleurs et leur proximité de la matière, l'auteur remarque ainsi qu'il est illusoire de croire que les tâches industrielles s'accordent à des facultés naturellement présentes chez les ouvriers. Ici, la considération mentionnée plus haut, concernant le fait que la production soit devenue une fin en soi, prend tout son sens critique. Halbwachs écrit: «on peut, par une sélection mieux comprise, spécialiser progressivement les ouvriers dans les tâches qui mettent en valeur leurs dons naturels: mais comme cela ne peut être l'objet dernier de l'industrie, comme l'essentiel est malgré tout de produire, avec un outillage défini, il faut bien que l'ouvrier ... s'adapte à sa tâche, puisqu'on ne peut adapter sa tâche exactement à ses dispositions et à sa nature ${ }^{60}$.

Durkheim est évidemment conscient du problème évoqué, comme le montrent les passages de La division du travail social consacrés au caractère insatisfaisant des occupations les plus répétitives et monotones. Seulement, ainsi que nous l'avons vu, la crise, analysée en termes d'inadaptation de la fonction sociale d'une personne à ses prédispositions naturelles, est en principe surmontable, c'est-à-dire que l'harmonie peut être réinstaurée, tandis que, selon Halbwachs, l'idée que la société moderne est organisée en vue de la production invalide, en principe (on

59 Durkheim, La division du travail social, op. cit., pp. 268, 369, 370. Cette représentation est mise en rapport avec l'idée de «personne»: «l'homme n'acquiert cette qualité [d'être une personne] que dans la mesure où il y a en lui quelque chose qui est à lui, à lui seul et qui l'individualise, où il est plus qu'une simple incarnation du type générique de sa race et de son groupe» (ibid., p. 399). De Queiroz (op. cit., pp. 120-127) a montré comment la notion de personne est susceptible de briser, de l'intérieur, le dualisme individu/ social.

${ }^{60}$ Halbwachs, Matière et société, op. cit., p. 83. 
pourrait presque dire, a priori), la représentation d'une correspondance idéale entre position professionnelle et penchants personnels.

Une deuxième objection doit être émise. En faisant allusion à un passage de la République de Platon, Halbwachs remarque, dans l'Esquisse, que le point de vue de la particularité individuelle revient au «naturalisme des anciens», pour qui «chaque être a une fonction qui dérive non pas des circonstances, mais de sa nature même». Quelle est la réplique qu'appelle cette position, du point de vue de la recherche scientifique? En un premier temps, on accordera évidemment qu'il existe «des hommes de toute nature dans les groupes les plus divers de la société ». Mais d'un autre côté, «on ne peut pas dire si oui, ou non, les hommes règlent leur conduite d'après leurs tendances naturelles, puisque leur nature est entièrement remaniée et transformée par la vie sociale». Il s'avère encore que la question de la «nature humaine» est d'emblée «mal posée ${ }^{61}$.

Le thème de la «nature» des hommes concerne, chez Platon, les vertus et les vices, comme le courage ou l'avidité, mais on voit que Halbwachs n'hésite pas à l'appliquer à la problématique des métiers et de l'origine des inégalités professionnelles et sociales. Ses propos sont sobres: «on ne naît pas paysan, gros propriétaire, fermier, manœuvrier de la campagne, en ce sens qu'on porterait dès la naissance, dans son organisme, annoncés et préformés, tous les traits qui caractérisent les hommes qui exercent ces métiers. On ne naît pas non plus bourgeois, entrepreneur, avocat, magistrat, ni ouvrier de la grande industrie. La nature de l'employé, du petit fonctionnaire, du petit commerçant n'est pas un fruit poussé spontanément sur la plante humaine». Les dons prédisposant les individus à des emplois déterminés n'existent donc pas à l'état observable, «mais en revanche, les catégories sociales existent $\gg^{62}$ : tout ce que l'on peut constater, c'est que les hommes sont effectivement fonctionnaires, commerçants, paysans, etc.

Il apparaît à nouveau que la notion d' «individualité» conduit dans une voie sans issue; on n'a jamais rencontré d'homme isolé, ni non plus de disposition personnelle à l'état pur. Et s'il est hors de doute que nous naissons inégaux, selon nos facultés, c'est-à-dire que nous ne possédons pas tous les mêmes dons pour les mathématiques, les langues, la musique, les travaux manuels, etc. ${ }^{63}$, il n'en demeure pas moins que, d'après Halbwachs, l'idée de talent naturel ne doit intervenir dans l'investigation scientifique de la vie sociale, et ne saurait servir de base à la compréhension des phénomènes observés. Elle serait plutôt comme un concept limite à la fois nécessaire et dépourvu de fonction explicative. Comme la recherche a dans tous les cas affaire aux «hommes tels qu'ils se présentent, encadrés dans les formations sociales $»^{64}$, le partage nature individuelle/ influences sociales est, là encore, totalement inefficace.

${ }^{61}$ Halbwachs, Esquisse..., op. cit., p. 209.

62 Ibid., p. 210.

${ }_{63}$ Dans le cas contraire, il faudrait admettre que les hommes sont parfaitement éducables. Récemment, R. Sennett a traité de cette expérience irréductible que constitue la répartition inégale des talents (R. Sennett, Respect. De la dignité de l'homme dans un monde d'inégalité, Paris, Ed. A. Michel, 2003, pp. 79-115).

${ }^{64}$ Ibid., p. 209. 


\section{LES FAITS SOCIAUX SONT DES CHOSES, LES FAITS INDIVIDUELS NON PLUS}

Le deuxième chapitre des Règles s'ouvre par la phrase suivante, qui exprime le programme sociologique de Durkheim de façon pointue: «La première règle et la plus fondamentale est de considérer les faits sociaux comme des choses. ${ }^{65} \mathrm{Au}$ chapitre précédent, Durkheim a préalablement établi ce à quoi ce reconnaissent ces faits: ils ont pour caractéristique distinctive d'être contraignants et extérieurs à l'individu $u^{66}$. Or la règle mentionnée implique un aspect important, auquel nous voudrions nous intéresser ici, à savoir que les faits individuels, eux aussi, doivent être considérés comme des choses. En effet, elle n'a de sens que si l'on accorde aux seconds la même réalité qu'aux premiers. Un fait ne peut être extérieur à une illusion ou exercer une force contraignante sur une chimère.

Je voudrais discuter deux remarques de Halbwachs, glanées au hasard des lectures, qui me semblent impliquer une critique acerbe de cette vision. La première se trouve dans Les cadres sociaux de la mémoire, au chapitre consacré à la religion, la seconde, dans la contribution Les caractéristiques des classes moyennes.

Vers le milieu du paragraphe intitulé «La mémoire collective religieuse», Halbwachs décrit comment la mémoire du passé a tendance à disparaître des pratiques religieuses routinières. C'est que la plupart des actes pieux - aller à la messe, etc. - sont liés pour les croyants à des préoccupations du présent, plutôt que de véritablement évoquer des événements anciens. Commentant cette observation, Halbwachs remarque: «Sans doute le fidèle conserve bien dans sa mémoire certains grands faits que l'instruction religieuse lui a enseignés...: mais du fait seul qu'il y a repensé souvent, et que d'autres y ont repensé avec lui, ces notions de fait sont devenues des notions de choses. ${ }^{67}$ Suivant cette indication, la masse des fidèles constitue comme un groupe sans mémoire.

Puis, dans le second texte, parmi une série d'observations concernant le rapport entre l'employé ou le fonctionnaire, d'un côté, et les personnes auxquelles ceux-ci ont affaire dans leur travail, de l'autre, on trouve la réflexion suivante: «L'employé lui-même s'aperçoit que ce ne sont pas tout à fait des choses sur lesquelles il opère, mais que ce sont des hommes: il peut être poli ou impoli; il peut se mettre à la disposition des clients, leur rendre certains services, leur donner quelques conseils qui ne dépassent pas une petite compétence: il peut les aider à gagner du temps; cependant l'essentiel de leur activité est de les traiter comme des choses ${ }^{68}$.

65 Durkheim, Les règles..., op. cit., p. 15.

${ }^{66}$ Une ambiguïté règne dans le texte de Durkheim: la contrainte extérieure fait-elle partie de la définition substantielle du fait social ou seulement de sa définition méthodologique? Les deux affirmations co-existent: «Est fait social toute manière de faire, fixée ou non, susceptible d'exercer sur l'individu une contrainte extérieure» (Durkheim, Les règles ..., op. cit., p. 14), et: «un fait social se reconnaît au pouvoir de coercition externe qu'il exerce ou qu'il est susceptible d'exercer sur les individus» (p. 11).

${ }^{67}$ M. Halbwachs, Les cadres sociaux de la mémoire, Postface de G. Namer, Paris, Ed. A. Michel, coll. Bibliothèque de l'Evolution de l'Humanité, 1994 [1925], p. 200 (nous soulignons).

${ }_{68}$ M. Halbwachs, Les caractéristiques des classes moyennes [1939], in Classes sociales et morphologie, op. cit., p. 107 (nous soulignons). 
Il ne s'agit pas ici d'affirmer que les phrases citées doivent s'entendre comme des critiques à la fois délibérées et sournoises, presque moqueuses, de la pensée de Durkheim. Est-il cependant illégitime d'y voir, en les prenant au pied de la lettre, des objections virtuelles à sa méthode?

Dans une telle perspective, qui pousse jusqu'à l'extrême la confrontation Halbwachs/ Durkheim, les réflexions mentionnées suggèrent que les Règles, loin de permettre de substituer, aux prénotions, des faits réels, ne représentent qu'un prolongement des premières. En vérité, traiter des «faits» ou des «hommes» comme des choses, c'est ce que nous faisons d'ordinaire: ou bien parce que, absorbés par nos soucis de tous les jours, nous sommes incapables d'écouter les récits d'autrefois, ou bien parce que les instructions bureaucratiques nous enjoignent d'exécuter des actes techniques «sans grande réflexion et sans réelle initiative $»^{69}$. Il faut considérer les faits sociaux comme des choses, écrit Durkheim; mais d'une certaine manière, le fonctionnaire accomplit la même opération lorsqu'il classe les personnes dans différentes catégories.

Autrement dit, les faits et les hommes se montrent à nous sous la forme de «choses» parce que nous sommes prisonniers d'habitudes ou de prescriptions qui nous empêchent d'entrer, avec eux, en un rapport de reconnaissance. Mais s'ils se présentent ainsi, c'est à l'évidence que les individus sont des «choses » à euxmêmes, comme ils sont reclus dans leur quotidien. La règle entérine le préjugé par excellence caractérisant nos représentations spontanées.

Evidemment, le précepte méthodologique dont il est ici question n'épuise pas la sociologie durkheimienne. Il n'empêche que les phrases de Halbwachs attirent l'attention sur un problème que Durkheim n'a su résoudre entièrement. «Le psychologue qui commence à s'enfermer dans son moi n'en peut plus sortir pour retrouver le non-moi », écrit ce dernier dans La division du travail social ${ }^{70}$. Ceci est vrai; mais il n'est pas certain qu'afin de dépasser l'erreur du psychologue, que partagent selon Durkheim les «utilitaristes», il faille opposer, à un individu demeurant enfermé, les formes sociales. Au contraire, tout indique que la confusion ne s'en trouve que reconduite.

Dans le repli sur soi-même, déconnecté de toute réalité extérieure - «il y moi, et il y a tout le reste» - l'individu ne peut trouver qu'un objet vide; et ceci se reflète exactement dans le fait que le monde des hommes prend à son tour l'aspect d'une affaire dénuée de vie. De ce point de vue, on peut affirmer que les pages centrales du Suicide, où Durkheim décrit la terrible solitude des hommes au sein de la société moderne ${ }^{71}$, représentent, non pas une mise en œuvre, mais une saisissante illustration de la vision du monde que véhicule sa méthode. En démantelant le dualisme société/ individu, Halbwachs combat, en dernière analyse, une forme de nihilisme sociologique qui risque de découler des concepts durkheimiens.

\footnotetext{
69 Ibid., p. 106.

7o Durkheim, La division du travail social, op. cit., p. 263.

${ }_{71}$ Durkheim, Le suicide, op. cit., pp. 222-233.
} 


\section{CONCLUSION}

«Peu de gens, actuellement, affirmeraient que leur existence psychique naît par génération spontanée, indépendamment des conditions sociales et des influences de l'environnement. Et pourtant, nous faisons exactement comme si le psychisme possédait une vie intérieure bien à lui » ${ }^{72}$. Ainsi que nous l'avons vu sur la base d'une analyse de textes de Halbwachs, ces phrases, écrites par R. Sennett, pourraient bien s'appliquer à l'œuvre de Durkheim. Le premier s'en prend à la représentation inhérente à la théorie durkheimienne selon laquelle l'individu possède une nature sui generis, et il en critique tous les aspects, à savoir l'idée du caractère tantôt autarcique tantôt insatiable et des dons innés de chaque personne, ainsi que celle du repli de l'homme sur lui-même. Il fait comprendre que c'est justement en expulsant l'individu en dehors d'elle-même que la sociologie crée une idée d'humanité abstraite.

On pourrait poursuivre et approfondir ces considérations en plusieurs directions. Par exemple, il vaudrait la peine de se demander comment Durkheim contribue à alimenter une représentation traditionnelle de l'individu, celle-là même qu'il entend réfuter, lorsqu'il est par exemple question chez lui de l'égoïsme et de l'illimitation des besoins. Autrement dit, le rapport de Durkheim aux différents courants philosophiques, y compris ceux auxquels il semble réfractaire, doit être reconsidéré.

En aval, il faudrait analyser comment la conception durkheimienne de l'individu a influencé la sociologie ultérieure et dans quelle mesure elle continue de le faire ${ }^{73}$.

Mais une autre question mériterait également d'être posée, et celle-ci s'adresse à Halbwachs.

Il est possible d'affirmer que Halbwachs achève, d'une certaine façon, la démarche de Durkheim consistant à établir la sociologie comme discipline autonome. Débarrassée de spéculations relatives à la constitution de l'individualité, la science sociologique semble dès lors être édifiée sur des fondements plus sûrs. Avec la disparition de l'individu pur, seul reste, l'homme tel qu'il vit au sein de la société.

Cependant, le problème est celui de savoir si le dépassement de la conception dualiste peut vraiment être mené jusqu'au bout ${ }^{74}$. C'est le livre posthume de Halbwachs, La mémoire collective, qui illustre le mieux la difficulté envisagée. La représentation de deux principes antinomiques s'y trouve reconduite sous une forme renouvelée, et les termes opposés se nomment désormais, le «clair» et l' «obscur».

Clersé, Université de Lille 1,

Faculté des Sciences économiques et Sociales

${ }_{72}$ R. Sennett, Les tyrannies de l' intimité, trad. de A. Berman et R. Folkman, Paris, Ed. du Seuil, 1979, p. 12.

${ }_{73}$ C. Arnsperger, Mauss et l'éthique du don: les enjeux d' un altruisme méthodologique, in «Les Cahiers du Mauss », 15, 2000, pp. 99-132, a récemment souligné la difficulté qu'éprouve la sociologie à faire «sortir» l'individu « du soi ».

${ }^{74}$ Il me semble indispensable, afin de pouvoir comprendre le cheminement de la jeune sociologie, de réexaminer le problème du dualisme d'un point de vue philosophique. On peut pour cela partir de F. Worms, Introduction à Matière et Mémoire de Bergson, suivie d'une brève introduction aux autres livres de Bergson, Paris, PUF, 1997, collection Les grands livres de la philosophie. 